\title{
Cellobionic acid inhibition of cellobiohydrolase I and cellobiose dehydrogenase
}

Amanda Hildebrand ${ }^{1}$, J. Bennett Addison ${ }^{2}$, Takao Kasuga ${ }^{3,4}$, and Zhiliang Fan ${ }^{1 *}$

${ }^{1}$ Department of Biological and Agricultural Engineering, University of California, Davis, One Shields Avenue, Davis, CA 95616, United States of America;

${ }^{2}$ Nuclear Magnetic Resonance Facility, University of California, Davis, One Shields Avenue, Davis, CA 95616, United States of America;

${ }^{3}$ Department of Plant Pathology, University of California, Davis, One Shields Avenue, Davis, CA 95616, United States of America;

${ }^{4}$ United States Department of Agriculture-Agricultural Research Service, Davis, CA 95616, United States of America

Corresponding author: Zhiliang Fan, Tel: 530-754-0317; Fax: 530-752-2640;

E-mail: jzfan@ucdavis.edu

\section{Abstract:}

End-product inhibition by cellobiose and glucose is a rate-limiting factor in cellulose hydrolysis by cellulases. While cellobiose and glucose inhibition have been extensively investigated, cellobionate inhibition has been minimally studied despite the discovery that accessory proteins such as cellobiose dehydrogenase $(\mathrm{CDH})$ work synergistically with cellulases to form aldonic acids. The fraction of equilibrated cellobionate in the cellobiono- $\delta$-lactone form was determined by NMR over the range of $\mathrm{pH} 4$ to 8 . To better improve the understanding of cellobiono- $\delta$ lactone and cellobionate inhibition of cellulases, we investigate the inhibition of Trichoderma reesei cellobiohydrolase $\mathrm{I}(\mathrm{CBHI})$, one of the most extensively studied $\mathrm{CBHs}$, and Neurospora 
crassa $\mathrm{CDH}$ by equilibrated cellobionate. The well-characterized inhibitor cellobiose is evaluated in parallel for comparison. The mechanisms of inhibition are determined for both inhibitors and the kinetic parameters compared using a Michaelis-Menten inhibition model. Cellobiose and cellobionate inhibition of $\mathrm{CBHI}$ is extended to Avicel hydrolysis, along with an investigation of those inhibitors on Accelerase 1500, a commercial cellulase preparation.

\section{Introduction:}

Due to its low cost and widespread abundance, lignocellulosic biomass is an attractive resource for renewable fuel and chemical production $[1,2]$. The primary obstacle to commercialization is the lack of a low cost processing technology, mainly due to the recalcitrance of cellulose. The steps involved in overcoming the recalcitrance (pretreatment, cellulase production, and enzymatic hydrolysis) account for approximately $40 \%$ of the total processing costs $[3,4]$.

Hydrolysis of cellulose is achieved by a cellulolytic system of enzymes comprised of three main classes of enzymes: cellobiohydrolases (CBHs), endoglucanases (EGs), and $\beta$ glucosidases (BGLs). EGs hydrolyze cellulose internally, while CBHs hydrolyze cellulose at the reducing and non-reducing ends, liberating cellobiose in the process. BGLs hydrolyze cellobiose to glucose, which can be fermented to fuels and chemicals. End-product inhibition by cellobiose and glucose is a rate-limiting factor, with cellobiose being the stronger inhibitor, and remains a primary technical challenge in the process of enzymatic hydrolysis [5-8]. Relieving inhibition provides an opportunity to significantly improve cellulose hydrolysis rates and the overall processing cost.

One way to relieve end-product inhibition is to utilize cellobiose dehydrogenase (CDH), which is an important enzyme produced along with cellulases by some cellulolytic 
microorganisms and catalyzes the oxidization of cellobiose to cellobionic acid. With the enzymatic oxidation of cellobiose via $\mathrm{CDH}$, cellobiono- $\delta$-lactone is formed initially $[9,10]$. However, cellobiono- $\delta$-lactone is thermodynamically unfavorable with increasing $\mathrm{pH}$ in aqueous solution and spontaneously hydrolyzes to cellobionate. Furthermore, many cellulolytic fungi secrete lactonases which quickly hydrolyze cellobiono- $\delta$-lactone to cellobionate $[11,12]$. It is speculated that the biological roles of $\mathrm{CDH}$ are to prevent product inhibition during cellulose degradation by oxidizing cellobiose, and to produce hydroxyl radicals through a Fenton-type reaction that can initiate depolymerization in lignin degradation $[9,13,14]$. Recently, the role of $\mathrm{CDH}$ was modified with the discovery that it works synergistically with polysaccharide monooxygenase accessory proteins (glycoside hydrolase 61 protein family, GH61) in oxidative cellulose degradation [15-18]. In our lab, we have developed a novel process which combines the cellulase production and enzymatic hydrolysis into a single step, and produces oxidized cellobiose (cellobionate) as the final hydrolysis product directly from cellulose. The engineered $N$. crassa strain with multiple copies of BGLs deleted contains all of the cellulase enzymes necessary for cellulose hydrolysis to cellobiose, with $\mathrm{CDH}$ oxidization of cellobiose to cellobionate $[19,20]$. Studying the inhibition of cellulases and CDH by cellobionate is useful for optimizing the system [21]. While cellobiose inhibition has been extensively investigated, cellobionate inhibition has been minimally studied. A previous study showed that cellobiono- $\delta$ lactone inhibits total cellulase activity to a greater extent than the same concentration of cellobiose, whereas cellobionate has a weaker inhibition compared to cellobiose [18]. That same study showed that cellobionate does not inhibit BGL activity, but does not investigate cellobionate inhibition of the other individual cellulase components $(\mathrm{CBH}, \mathrm{EG}$, or $\mathrm{CDH})$. Another study evaluates the kinetics of lactobiono- $\delta$-lactone and lactobionate inhibition of CDH 
with lactose as the substrate, concluding that the lactone is more inhibitory than the practically non-inhibitory acid form [12]. Currently, no kinetic information is available in the literature for cellobiono- $\delta$-lactone or cellobionate inhibition of $\mathrm{CDH}$. The $\mathrm{K}_{\mathrm{m}}$ value of $\mathrm{CDH}$ for lactose is approximately 20-fold higher than the $\mathrm{K}_{\mathrm{m}}$ value for cellobiose [12], making it difficult to infer that product inhibition of lactose is kinetically similar to product inhibition of cellobiose. To better improve the understanding of cellobiono- $\delta$-lactone and cellobionate inhibition of cellulases, we investigate the inhibition of Trichoderma reesei $\mathrm{CBHI}$, one of the most extensively studied CBHs, and the $N$. crassa $\mathrm{CDH}$. The well-studied cellobiose inhibitor is characterized in parallel for comparison. The mechanisms of inhibition are determined for both inhibitors and the kinetic parameters compared using a Michaelis-Menten inhibition model. The inhibition of $\mathrm{CBHI}$ by cellobiose or cellobionate using Avicel as a substrate is also evaluated, as well as the inhibition of Accelerase 1500, a commercial cellulase cocktail. To our knowledge, this study provides the first direct comparison of cellobiose and cellobionate inhibition of CBHI and the first kinetic evaluation of cellobionate inhibition of $\mathrm{CDH}$.

\section{Materials and Methods:}

\section{Reagents:}

CBHI from T. reesei, $p$-nitrophenyl- $\beta$-D-lactopyranoside (pNPL), and cellobiose were obtained from Sigma Aldrich (St. Louis, MO). CDH was obtained by a recombinant Pichia pastoris strain expressing CDHI from $N$. crassa as described previously [22]. Cellobionate was synthesized in our laboratory according to a previously established method as follows [23]. $3.8 \mathrm{~g}$ of cellobiose was dissolved in $20 \mathrm{~mL}$ of deionized water and heated at $55^{\circ} \mathrm{C}$ for 10 minutes with stirring until completely dissolved. The solution was added to $50 \mathrm{~mL}$ of methanol. A solution of $5.7 \mathrm{~g}$ of iodine in $5 \mathrm{~mL}$ of methanol was added to the cellobiose solution and heated at $40{ }^{\circ} \mathrm{C}$ for 15 
minutes with stirring. At room temperature and with stirring, $100 \mathrm{~mL}$ of $4 \%(\mathrm{w} / \mathrm{v})$ potassium hydroxide-methanol solution was slowly added to the cellobiose-iodine solution, forming a sticky yellow precipitate. Another $100 \mathrm{~mL}$ of $4 \%(\mathrm{w} / \mathrm{v})$ potassium hydroxide-methanol solution was added slowly, and the solution was stirred for an additional 30 minutes at room temperature. The cloudy top solution containing un-converted cellobiose was decanted from the yellow precipitate. The precipitate was dissolved in $5 \mathrm{~mL}$ of deionized water, to which another $100 \mathrm{~mL}$ of methanol was added. A white precipitate forms and settles quickly. Methanol is removed by centrifugation $(6000 \mathrm{~g}, 20$ minute) and decanted. The potassium cellobionate precipitate was washed with $50 \mathrm{~mL}$ of methanol (three times) and tested for purity by HPLC.

\section{NMR analysis:}

${ }^{1} \mathrm{H}$ NMR spectra were collected at $35^{\circ} \mathrm{C}$ using the standard $90^{\circ}$ pulse and acquire sequence on an $800 \mathrm{MHz}$ Bruker Avance III spectrometer equipped with a $5 \mathrm{~mm}$ Bruker HCN tripleresonance cryoprobe. NMR samples contained $5 \mathrm{mM}$ cellobionate in buffer and $99.96 \% \mathrm{D}_{2} \mathrm{O}$ and were allowed to equilibrate for 72 hours prior to analysis. For $\mathrm{pH} 4$ and 5, $10 \mathrm{mM}$ sodium citrate buffer was used. For $\mathrm{pH} \mathrm{6,7,} \mathrm{and} \mathrm{8,} 50 \mathrm{mM}$ sodium phosphate buffer was used. The desired $\mathrm{pD}$ was confirmed by a Metler Toledo glass $\mathrm{pH}$ meter, with the $\mathrm{pH}$ reading converted to pD by adding 0.4 [24]. NMR acquisition parameters were as follows: 32 scan averages, $11 \mathrm{ppm}$ spectral width centered about the HOD resonance, 61618 points collected, 3.5 second acquisition time and a 3 second recycle delay for a total of 6.5 seconds repetition rate. The raw freeinduction decay (FID) was multiplied by a $0.1 \mathrm{~Hz}$ exponential line broadening function before Fourier transform. Each spectrum was phased and baseline corrected, and chemical shifts were referenced by setting the internal anemic hydrogen of $\alpha$-cellobiose to $5.23 \mathrm{ppm}$ [10]. Peak integrations were used to measure the cellobiono- $\delta$-lactone to cellobionate ratios. 


\section{Inhibition studies:}

Inhibition effects of cellobiose and cellobionate on CBHI were evaluated using pNPL as a substrate in a previously established enzymatic assay [25]. Final concentrations of reactants were $2.2 \mathrm{mM}(1 \mathrm{mg} / \mathrm{mL}) \mathrm{pNPL}, 0.05 \mathrm{U} / \mathrm{mL}$ of CBHI, and $0-5 \mathrm{mM}$ of inhibitor in a total volume of $180 \mu \mathrm{L}$. Solutions containing cellobiose or cellobionate inhibitors were allowed to equilibrate for at least 48 hours prior to analysis. The enzymatic reaction was initiated by the addition of enzyme and stopped after 15 minutes by the addition of $120 \mu \mathrm{L}$ of $1 \mathrm{M} \mathrm{NaOH}$. The absorbance at $405 \mathrm{~nm}$ was compared to a standard curve of p-nitrophenol to determine the rate of release of p-nitrophenol. One unit of enzyme activity corresponds to the release of $1 \mu$ mol of p-nitrophenol per minute. The assay was carried out in $50 \mathrm{mM}$ sodium citrate buffer at $\mathrm{pH} 4$ or $\mathrm{pH} 6$ to determine the effect of the presence or absence of the lactone.

The analysis was repeated using an Accelerase 1500 cellulase cocktail at a final concentration of $0.05 \mathrm{U} / \mathrm{mL}$ (activity determined on pNPL according to the assay conditions described above). The assay was carried out in $50 \mathrm{mM}$ sodium citrate buffer at $\mathrm{pH}$, the standard assay $\mathrm{pH}$ according to the previously established method [25].

The inhibition effect of cellobionate on $\mathrm{CDH}$ was evaluated using cellobiose as a substrate. Final concentrations of reactants were $0.015 \mathrm{U} / \mathrm{mL} \mathrm{CDH}, 30 \mathrm{mM}$ cellobiose, $0.3 \mathrm{mM}$ 2,6dichlorophenolindophenol (DCPIP) and 0 - $20 \mathrm{mM}$ of cellobionate in a total volume of $200 \mu \mathrm{L}$. $\mathrm{CDH}$ activity was determined by following the decrease in absorbance of DCPIP at $520 \mathrm{~nm}$, as described previously, with one unit of enzyme activity corresponding to the reduction of $1 \mu$ mol of DCPIP per minute [26]. The assay was conducted in $100 \mathrm{mM}$ sodium citrate buffer at $\mathrm{pH} 4$ or pH 6 to determine the effect of the presence of the lactone. 


\section{Kinetic analysis:}

To determine the mechanism and kinetic parameters of cellobiose and cellobionate inhibition of CBHI, velocity versus substrate curves were generated for $\mathrm{CBHI}$ with various concentrations of pNPL (0.05 mM - $4 \mathrm{mM})$ and various inhibitor concentrations resulting in $20-80 \%$ inhibition $(0.1-0.5 \mathrm{mM}$ for cellobiose and $0.5-2.5 \mathrm{mM}$ for cellobionate). The assay was performed in 50 $\mathrm{mM}$ sodium citrate buffer at $\mathrm{pH}$ 5. Assay conditions were maintained so that the total $\mathrm{p}$ nitrophenol released was less than $5 \%$ of the initial substrate concentration. Lineweaver-Burk plots were generated to determine the mechanism of inhibition. The kinetic parameters were determined using non-linear regression (PolyMath), fitting the data to the appropriate MichaelisMenten equation describing the mechanism observed from the Lineweaver-Burk plot. The mechanism and kinetic parameters of cellobionate inhibition of $\mathrm{CDH}$ were similarly determined, with the cellobiose substrate ranging from $0.05-10 \mathrm{mM}$ and inhibitor concentration ranging from $0-10 \mathrm{mM}$. For kinetic analysis of $\mathrm{CDH}$, the assay was conducted at $\mathrm{pH} 5$ in $100 \mathrm{mM}$ sodium acetate buffer.

\section{Inhibition of equilibrated cellobionate solution on CBHI using Avicel as the substrate:}

Hydrolysis reactions contained $50 \mathrm{mM}$ sodium citrate buffer (pH 5.0), $20 \mathrm{~g} / \mathrm{L}$ Avicel, and 10 $\mathrm{mg} / \mathrm{mL}$ CBHI. The extent of hydrolysis with and without $2.5 \mathrm{mM}$ of inhibitor (cellobiose or cellobionate) was evaluated by monitoring the release of reducing sugars using the dinitrosalicylic acid (DNS) method [27]. Samples were taken at various time points and centrifuged to remove unhydrolyzed Avicel. After 15 minutes of incubation at $37^{\circ} \mathrm{C}$, DNS reagent $(0.01 \mathrm{~g}$ DNS, $3 \mathrm{~g}$ sodium potassium tartrate, $4 \mathrm{~mL} 1 \mathrm{M} \mathrm{NaOH}$, with a total volume of 10 $\mu \mathrm{L}$ with DI water) was added to the sample and incubated in a thermocycler for 5 minutes at 95 ${ }^{\circ} \mathrm{C}$, followed by 1 minute at $4{ }^{\circ} \mathrm{C}$. The absorbance was measured at $540 \mathrm{~nm}$ and compared to 
glucose standards $(0-1.0 \mathrm{~g} / \mathrm{L})$. One unit of activity corresponds to the release of $1 \mu \mathrm{mol}$ of reducing sugar per minute.

\section{Results:}

We explore the inhibition effects of equilibrated mixtures of cellobionate on CBHI and CDH activity. In studies involving CBHI, cellobiose is also evaluated as a point of comparison. The mechanisms of inhibition of the equilibrated mixtures on both enzymes are evaluated and the kinetic parameters determined using a Michaelis-Menten model.

\section{NMR analysis:}

Equilibrium mixtures of cellobionate were analyzed by NMR to determine the relative ratios of cellobiono- $\delta$-lactone and acid in the mixture at different $\mathrm{pH}$. The chemical structures of the lactone and acid forms are given in Figure 1, and the NMR spectra for the $\mathrm{pH}$ range tested is shown in Figure 2. The protons corresponding to the relevant peaks are coded with a colored symbol as indicated in Figures 1 and 2. As determined previously, the chemical shifts at 4.58 ppm and $4.28 \mathrm{ppm}$ correspond to the internal anomeric proton (red square) and C-2 proton (purple circle) of cellobiono- $\delta$-lactone, respectively [10]. The chemical shift for the internal anomeric proton (blue diamond) of cellobionate is shifted up field to $4.63 \mathrm{ppm}$. The chemical shift for the C-2 proton (green triangle) of cellobionate ranges from $4.29-4.15$ ppm, depending on the ratio of protonated to unprotonated acid as dictated by the $\mathrm{pH}[10]$. The relative ratios of cellobiono- $\delta$-lactone and cellobionate can be determined by comparing the peak areas of a given proton [28], as given in Table 1. The calculated relative amounts of cellobiono- $\delta$-lactone and cellobionic acid align with those determined for glucono- $\delta$-lactone and gluconic acid $[29,30]$, indicating that above $\mathrm{pH} 5$ the equilibrium tends to shift toward aldonic acids [15]. 


\section{Inhibition of cellulases by cellobiose and cellobionate:}

It is important to understand the effect of equilibrated cellobionate mixtures on cellulases in order to develop appropriate end-product inhibition relief strategies. Figure 3 shows the inhibition of CBHI by cellobiose or cellobionate using pNPL as a model substrate. Figure 3A shows that the extent of inhibition of $\mathrm{CBHI}$ by cellobionate is similar at $\mathrm{pH} 4$ where the lactone form constitutes $5.4 \%$ of the equilibrated solution compared to $\mathrm{pH} 6$ where only the cellobionate form exists. The data suggest that the small amount of lactone present does not significantly impact the results. Figure $3 \mathrm{~B}$ shows the inhibition of $\mathrm{CBHI}$ by cellobiose. $\mathrm{CBHI}$ is inhibited to a greater extent by cellobiose compared to the same concentration of an equilibrated solution of cellobionate. Contrary to inhibition of $\mathrm{CBHI}$, inhibition of $\mathrm{CDH}$ by an equilibrated solution of cellobionate at pH 4 and $\mathrm{pH} 6$ show drastically differing results (Figure 3C). At pH 6, no inhibition is observed for cellobionate concentrations up to $20 \mathrm{mM}$. In contrast, at pH 4 where lactone is present, $\mathrm{CDH}$ is inhibited. This data suggests that the inhibition observed at $\mathrm{pH} 4$ is due to the inhibitory effect of the lactone. The inhibition effects of cellobiose and cellobionate on Accelerase 1500, a commercial preparation of $T$. reesei cellulases, were subsequently evaluated (Figure 3D). The results again show decreased inhibition for cellobionate compared to cellobiose. Accelerase 1500 was less inhibited for a given concentration of cellobiose or cellobionate compared to purified T. reesei $\mathrm{CBHI}$.

Next, the evaluation of cellobionate as an inhibitor was extended to Avicel, a real cellulosic substrate, for comparison to the model pNPL substrate. The hydrolysis of Avicel with CBHI with and without $2.5 \mathrm{mM}$ of inhibitor (cellobiose or cellobionate) is shown in Figure 4. Avicel is hydrolyzed to a greater extent without either inhibitor present. The presence of $2.5 \mathrm{mM}$ of 
cellobionate inhibits Avicel hydrolysis to a lesser extent than the same concentration of cellobiose, a result that agrees with the inhibition observed with pNPL as a substrate.

\section{Determination of kinetic parameters:}

To determine the mechanism of inhibition, the reaction velocity was determined for various concentrations of inhibitor and substrate for $\mathrm{CDH}$ and $\mathrm{CBHI}$. The double-reciprocal plots of the data, shown in Figure 5, depict a typical competitive inhibition mechanism for Michaelis-Menten kinetics. Based on the competitive inhibition Michaelis-Menten model (Equation 1), the kinetic parameters were calculated by non-linear regression and are listed in Table 2, where $\mathrm{V}_{\max }$ is the maximum reaction rate in $\mathrm{mM} / \mathrm{min}, \mathrm{S}$ is the substrate concentration in $\mathrm{mM}, \mathrm{K}_{\mathrm{m}}$ is the MichaelisMenten constant in $\mathrm{mM}$, I is the inhibitor concentration in $\mathrm{mM}$, and $\mathrm{K}_{\mathrm{i}}$ is the inhibition constant in $\mathrm{mM}$.

Equation $1 \quad v=\frac{V \max * S}{K_{m}\left(1+\frac{I}{K_{i}}\right)+S}$

The $\mathrm{K}_{\mathrm{m}}$ value of CBHI on pNPL was $1.05 \mathrm{mM}$. The $\mathrm{K}_{\mathrm{i}}$ values of cellobiose and cellobionate inhibition of CBHI were $0.05 \mathrm{mM}$ and $1.18 \mathrm{mM}$, respectively, indicting a 23-fold stronger inhibition by cellobiose than by cellobionate. The $\mathrm{K}_{\mathrm{m}}$ value of $\mathrm{CDH}$ with cellobiose as a substrate was $0.172 \mathrm{mM}$, and the $\mathrm{K}_{\mathrm{i}}$ value of cellobionate inhibition of $\mathrm{CDH}$ was $0.732 \mathrm{mM}$.

\section{Discussion:}

In this study, we seek to understand the inhibition effects of an equilibrated cellobionate solution on cellulases. Inhibition of CBHI by cellobionate was compared to inhibition by cellobiose. It was found that cellobionate is less inhibitory than cellobiose for CBHI, as well as the commercial cellulase preparation Accelerase 1500, when pNPL is used as a substrate. Similar 
results were found for $\mathrm{CBHI}$ hydrolysis of Avicel. Inhibition of $\mathrm{CDH}$ by cellobionate was also evaluated. No inhibition was observed when only the acid form was present, whereas an equilibrated mixture of cellobionate containing the lactone form does show inhibition. Inhibition of both $\mathrm{CBHI}$ and $\mathrm{CDH}$ were modeled according to Michaelis-Menten kinetics.

The NMR data presented here show that equilibrated cellobionate contains approximately $5.4 \%$ cellobiono- $\delta$-lactone at $\mathrm{pH} 4$. In contrast, at $\mathrm{pH} 6$ an equilibrated cellobionate solution contains only the acid form. At $\mathrm{pH} \mathrm{5,} \mathrm{which} \mathrm{is} \mathrm{the} \mathrm{optimal} \mathrm{pH}$ for cellulases, the equilibrated cellobionate contains approximately $1.9 \%$ cellobiono- $\delta$-lactone. Obvious inhibition of CBHI by cellobionate at $\mathrm{pH} 6$ was observed, which indicates that the cellobionate is inhibitory to CBHI activity in $100 \%$ acid form. Inhibition of $T$. reesei CBHI by a cellobionate solution at $\mathrm{pH} 4$ shows a similar degree of inhibition to a cellobionate solution at $\mathrm{pH}$ 6, implying that the presence of a small fraction of cellobiono- $\delta$-lactone does not change the extent of inhibition of the cellobionate solution on CBHI activity significantly.

In parallel experiments, cellobiose inhibition on $\mathrm{CBHI}$ was tested at the same concentration as cellobionate and showed much stronger inhibition. This result aligns with a previous study evaluating the effects of hydrolysis products on cellulase activity as a whole, which showed that cellobiose had a stronger inhibitory effect compared to cellobionate [18]. Additionally, the extent of inhibition of CBHI by cellobiose is similar at $\mathrm{pH} 4$ and $\mathrm{pH} 6$.

When Accelerase 1500, a commercial cellulase cocktail, is evaluated, cellobiose again shows stronger inhibition compared to cellobionate for a given inhibitor concentration. However, in contrast to purified CBHI, the magnitude of inhibition by either cellobiose or cellobionate decreases when the cellulase cocktail is used. This result is not surprising, since cellulase cocktails contain a variety of enzymes, including CBHIs, CBHIIs, EGs, and BGLs. Previous 
studies have shown that CBHI enzymes are inhibited by cellobiose to a greater extent than other cellulases [5-7]. Lastly, when cellobionate inhibition is compared to cellobiose inhibition using Avicel as a substrate, the rate and extent of hydrolysis is less impacted by the presence of cellobionate than by the presence of cellobiose, a result similar to that obtained when pNPL is used as the substrate.

To elucidate the mechanism of inhibition, the reaction rate was calculated for several substrate and inhibitor concentrations and double-reciprocal plots were generated as shown in Figure 5. The double-reciprocal plots depict a competitive inhibition mechanism. The data was fit to the Michaelis-Menten equation for competitive inhibition (Equation 1) using non-linear regression to determine the kinetic parameters, as shown in Table 2. The calculated $\mathrm{K}_{\mathrm{m}}$ value aligns with previous results in literature for $\mathrm{pNPL}$ and $T$. reesei $\mathrm{CBHI}$ [25], and the mechanism of inhibition and calculated $\mathrm{K}_{\mathrm{i}}$ value for cellobiose aligns with a similar study with the CBHI from Phanerochaete chrysosporum [31]. The $\mathrm{K}_{\mathrm{i}}$ value for cellobionate inhibition of CBHI was 23 times higher than that of cellobiose, indicating a much weaker inhibition effect. This result is supported by a study showing that cellobiose inhibition of CBHI by P. chryosporium is relieved in the presence of $\mathrm{CDH}$ and a redox mediator, which would convert the cellobiose to cellobiono$\delta$-lactone and cellobionate [31]. However, the same study shows that inhibition of CBHI from $T$. viride increases in the presence of $\mathrm{CDH}$ and a redox mediator. It appears that the effects of cellobiono- $\delta$-lactone and cellobionate on CBHI is dependent on the source of CBHI.

In contrast to cellobionate inhibition of CBHI from T. reesei, $\mathrm{CDH}$ from $N$. crassa does not appear to be inhibited by cellobionate. At $\mathrm{pH}$ 6, where cellobionate is present in only its acid form, no inhibition is observed for concentrations up to $20 \mathrm{mM}$. At $\mathrm{pH} 4$, where $5.4 \%$ of the cellobionate solution is in the lactone form, inhibition is observed. These results suggest that the 
cellobiono- $\delta$-lactone is significantly more inhibitory on $\mathrm{CDH}$ than cellobionate, which is quite different than its effect on CBHI. This result is similar to a previous study evaluating the effects of lactobionate and lactobiono- $\delta$-lactone inhibition of $\mathrm{CDH}$ when lactose is used as a substrate [12]. It is also important to note that the $\mathrm{K}_{\mathrm{m}}$ value of $\mathrm{CDH}$ with lactose is $2.4 \mathrm{mM}$ at $25^{\circ} \mathrm{C}$, compared to $0.12 \mathrm{mM}$ when cellobiose is the substrate [12]. Our calculated $\mathrm{K}_{\mathrm{m}}$ value of $0.17 \mathrm{mM}$ at $30{ }^{\circ} \mathrm{C}$ aligns well with the literature values of $0.12 \mathrm{mM}$ at $25^{\circ} \mathrm{C}$ and $0.28 \mathrm{mM}$ at $45^{\circ} \mathrm{C}$ [12]. While the acid form of cellobionate does not have an inhibitory effect on $\mathrm{CDH}$, our kinetic studies show that an equilibrated cellobionate solution (where a portion is in the lactone form), has a notable inhibitory effect. The calculated $\mathrm{K}_{\mathrm{i}}$ value for an equilibrated cellobionate solution is $0.73 \mathrm{mM}$. This indicates a much stronger inhibition as compared to an equilibrated lactobionate solution, where the authors report the $\mathrm{K}_{\mathrm{i}}$ values for an equilibrated lactobionic acid solution, and a calcium lactobionate solution to be $510 \mathrm{mM}$ and $1600 \mathrm{mM}$, respectively [12]. These values are much higher than the $\mathrm{K}_{\mathrm{i}}$ value we find for an equilibrated cellobionate solution on $\mathrm{CDH}$ using cellobiose as the substrate. It is difficult to draw comparisons since the cited study has an equilibriated mixture of lactobionic acid that contains $84 \%$ acid and $16 \%$ lactone, which is quite different than our relative ratio. In addition, they characterized a $\mathrm{CDH}$ from a different microorganism, which may have a different affinity to substrates and inhibitors.

In summary, this study shows that cellobionate inhibition is weaker than cellobiose inhibition for purified CBHI or a commercial cellulase blend using the model pNPL substrate, as well as for purified CBHI using Avicel as a substrate. Our data show that the $\mathrm{K}_{\mathrm{i}}$ value for cellobionate is approximately 23 times higher than that for cellobiose for CBHI from T. reesei, indicating a much weaker inhibition effect. Since $T$. reesei's $\mathrm{CBHI}$ is considered one of the workhorse enzymes for cellulose hydrolysis, and CBHs are the class of cellulases most strongly 
inhibited by cellobiose [5-7], cellobiose inhibition relief strategies are important for improving the overall rate of enzymatic conversion of cellulose [32]. Our data show that oxidation of cellobiose to cellobionate will likely improve conversion rates through such inhibition relief. It is noteworthy that cellobionate can be an alternative substrate for fuels and chemicals production via fermentation. Ethanol production from cellobionate can be achieved by engineering $E$. coli to express BGL or by adding exogenous BGL to hydrolyze cellobionate into equimolar amounts of glucose and gluconate. Both of the hydrolysis products from cellobionate, glucose and gluconate, can be used efficiently by E. coli KO11 to produce ethanol and acetic acid [33,34]. Alternatively, E. coli can directly consume cellobionate as the carbon source for isobutanol production; Saccharomyces cerevisiae has been engineered to use cellobionate for ethanol production $[21,35]$. Furthermore, equilibrated solutions of cellobionate containing cellobiono- $\delta$ lactone have significant inhibition of $\mathrm{CDH}$, highlighting the importance of lactonases in whole cellulolytic systems $[11,18]$. The extent to which cellulases other than $\mathrm{CBHI}$ and $\mathrm{CDH}$ are inhibited by cellobionate is beyond the scope of this study. However, the fact that CBHIs are considered the workhorse enzyme of cellulases suggests that relieving inhibition of this enzyme can potentially improve hydrolysis rates of cellulose for the complete cellulolytic system. In support of this hypothesis, we show that Accellerase 1500, a commercial cellulase preparation, is inhibited to a lesser extent by cellobionate than by cellobiose.

\section{Conclusions}

This study describes the inhibitory effect of equilibrated cellobionate on cellulases. Compared to cellobiose, equilibrated cellobionate is less inhibitory for CBHI and Accelerase 1500 when pNPL is used as a substrate. Similarly, Avicel hydrolysis by CBHI is inhibited to a lesser extent by equilibrated cellobionate compared to inhibition by cellobiose. $\mathrm{CDH}$ is inhibited when the 
lactone form is present in the equilibrated cellobionate solution. No inhibition of $\mathrm{CDH}$ is observed when only the acid form is present. While additional studies can further elucidate the mechanisms behind inhibition reduction, this study demonstrates the potential of converting cellobiose to cellobionate to partially relieve product inhibition by cellobiose and provides the motivation for continued research in this area.

\section{Acknowledgements:}

This project was supported by Agriculture and Food Research Initiative Competitive Grant No. 2011-67009-20060 from the USDA National Institute of Food and Agriculture, as well as EPA Star and Cota-Robles fellowships awarded to Amanda Hildebrand. NSF DBIO-722538 provided funding for the $800 \mathrm{MHz}$ Bruker Avance III NMR spectrometer.

\section{References:}

[1] L.R. Lynd, J. Cushman, R. Nichols, C. Wyman, Fuel ethanol from cellulosic biomass, Science (80-. ). 251 (1991) 1318-1323.

[2] D. Humbird, R. Davis, L. Tao, C. Kinchin, D. Hsu, A. Aden, Process Design and Economics for Biochemical Conversion of Lignocellulosic Biomass to Ethanol Process Design and Economics for Biochemical Conversion of Lignocellulosic Biomass to Ethanol, NREL/TP-5100-47764. (2011).

[3] R. Wooley, M. Ruth, J. Sheehan, K. Ibsen, H. Majdeski, A. Galvez, Lignocellulosic Biomass to Ethanol Process Design and Economics Utilizing Co-Current Dilute Acid Prehydrolysis and Enzymatic Hydrolysis Current and Futuristic Scenarios, 1999.

[4] M.E. Himmel, S.-Y. Ding, D.K. Johnson, W.S. Adney, M.R. Nimlos, J.W. Brady, et al., Biomass recalcitrance: engineering plants and enzymes for biofuels production, Science (80-. ). 315 (2007) 804-807. doi:10.1126/science.1137016.

[5] H. Teugjas, P. Väljamäe, Product inhibition of cellulases studied with 14C-labeled 
cellulose substrates., Biotechnol. Biofuels. 6 (2013) 104. doi:10.1186/1754-6834-6-104.

[6] L. Murphy, C. Bohlin, M.J. Baumann, S.N. Olsen, T.H. Sørensen, L. Anderson, et al., Product inhibition of five Hypocrea jecorina cellulases., Enzyme Microb. Technol. 52 (2013) 163-9. doi:10.1016/j.enzmictec.2013.01.002.

[7] M. Gruno, P. Väljamäe, G. Pettersson, G. Johansson, Inhibition of the Trichoderma reesei cellulases by cellobiose is strongly dependent on the nature of the substrate., Biotechnol. Bioeng. 86 (2004) 503-11. doi:10.1002/bit.10838.

[8] P. Andrić, A.S. Meyer, P. a Jensen, K. Dam-Johansen, Reactor design for minimizing product inhibition during enzymatic lignocellulose hydrolysis: I. Significance and mechanism of cellobiose and glucose inhibition on cellulolytic enzymes., Biotechnol. Adv. 28 (2010) 308-24. doi:10.1016/j.biotechadv.2010.01.003.

[9] G. Henriksson, G. Johansson, G. Pettersson, A critical review of cellobiose dehydrogenases, J. Biotechnol. 78 (2000) 93-113. doi:10.1016/s0168-1656(00)00206-6.

[10] C.W. Higham, D. Gordon-Smith, C.E. Dempsey, P.M. Wood, Direct 1H NMR evidence for conversion of $\beta$-d-cellobiose to cellobionolactone by cellobiose dehydrogenase from Phanerochaete chrysosporium, FEBS Lett. 351 (1994) 128-132. doi:10.1016/00145793(94)00847-7.

[11] W.T. Beeson, A.T. Iavarone, C.D. Hausmann, J.H.D. Cate, M. a Marletta, Extracellular aldonolactonase from Myceliophthora thermophila., Appl. Environ. Microbiol. 77 (2011) 650-6. doi:10.1128/AEM.01922-10.

[12] U. Baminger, S.S. Subramaniam, V. Renganathan, D. Haltrich, Purification and characterization of cellobiose dehydrogenase from the plant pathogen Sclerotium (Athelia) rolfsii, Appl Env. Microbiol. 67 (2001) 1766-1774. doi:10.1128/AEM.67.4.1766-1774.2001.

[13] L. Hildén, G. Johansson, G. Pettersson, J. Li, P. Ljungquist, G. Henriksson, Do the extracellular enzymes cellobiose dehydrogenase and manganese peroxidase form a pathway in lignin biodegradation?, FEBS Lett. 477 (2000) 79-83. doi:10.1016/S00145793(00)01757-9.

[14] S.D. Mansfield, E. De Jong, J.N. Saddler, Cellobiose dehydrogenase, an active agent in cellulose depolymerization, Appl. Environ. Microbiol. 63 (1997) 3804-3809. 
[15] D. Cannella, C.-W.C. Hsieh, C. Felby, H. Jørgensen, T.P. Pdf, I. Article, et al., Production and effect of aldonic acids during enzymatic hydrolysis oflignocellulose at high dry matter content, Biotechnol. Biofuels. 5 (2012) 20. doi:10.1186/1754-6834-5-26.

[16] W.T. Beeson, C.M. Phillips, J.H. Cate, M.A. Marletta, Oxidative cleavage of cellulose by fungal copper-dependent polysaccharide monooxygenases, J. Am. Chem. Soc. 134 (2012) 890-892. doi:10.1021/ja210657t.

[17] C.M. Phillips, W.T. Beeson, J.H. Cate, M.A. Marletta, Cellobiose dehydrogenase and a copper-dependent polysaccharide monooxygenase potentiate cellulose degradation by Neurospora crassa, ACS Chem Biol. 6 (2011) 1399-1406. doi:10.1021/cb200351y.

[18] E. Bruchmann, H. Schach, H. Graf, Role and Properties of Lactonases in a Cellulase System, Biotechnol. Appl. Biochem. 9 (1987) 146-159.

[19] A. Hildebrand, E. Szewczyk, H. Lin, T. Kasuga, Z. Fan, Engineering Neurospora crassa for Improved Cellobiose and Cellobionate Production, Appl. Environ. Microbiol. 81 (2015) 597-603. doi:10.1128/AEM.02885-14.

[20] A. Hildebrand, T. Kasuga, Z. Fan, Production of Cellobionate from Cellulose Using an Engineered Neurospora crassa Strain with Laccase and Redox Mediator Addition, PLoS One. 10 (2015) e0123006. doi:10.1371/journal.pone.0123006.

[21] X. Li, K. Chomvong, V.Y. Yu, J.M. Liang, Y. Lin, J.H.D. Cate, Cellobionic acid utilization: from Neurospora crassa to Saccharomyces cerevisiae, Biotechnol. Biofuels. (2015) 1-9. doi:10.1186/s13068-015-0303-2.

[22] R. Zhang, C. Xu, Q. Shen, T. Kasuga, W. Wu, E. Szewczyk, et al., Characterization of two cellobiose dehydrogenases and comparison of their contributions to total activity in Neurospora crassa, Int. Biodeterior. Biodegradation. 82 (2013) 24-32. doi:10.1016/j.ibiod.2013.03.017.

[23] J.W. Green, N.S. Thompson, I.A. Pearl, Study of the carbohydrate peeling and stopping reactions under the conditions of oxygen-alkali pulping, 1976.

[24] P.K. Glasoe, F.A. Long, Use of Glass Electrodes to Measure Acidities in Deuterium Oxide, J. Phys. Chem. 64 (1960) 188-190.

[25] M. V. Deshpande, K.-E. Eriksson, L. Göran Pettersson, An assay for selective 
determination of exo-1,4,- $\beta$-glucanases in a mixture of cellulolytic enzymes, Anal. Biochem. 138 (1984) 481-487. doi:10.1016/0003-2697(84)90843-1.

[26] U. Baminger, B. Nidetzky, K.D. Kulbe, D. Haltrich, A simple assay for measuring cellobiose dehydrogenase activity in the presence of laccase, 35 (1999) 253-259.

[27] B.C. King, M.K. Donnelly, G.C. Bergstrom, L.P. Walker, D.M. Gibson, An optimized microplate assay system for quantitative evaluation of plant cell wall-degrading enzyme activity of fungal culture extracts., Biotechnol. Bioeng. 102 (2009) 1033-44. doi:10.1002/bit.22151.

[28] S.K. Bharti, R. Roy, Quantitative 1H NMR spectroscopy, TrAC - Trends Anal. Chem. 35 (2012) 5-26. doi:10.1016/j.trac.2012.02.007.

[29] Z. Zhang, P. Gibson, S.B. Clark, G. Tian, P.L. Zanonato, L. Rao, Lactonization and Protonation of Gluconic Acid: A Thermodynamic and Kinetic Study by Potentiometry, NMR and ESI-MS, J. Solution Chem. 36 (2007) 1187-1200. doi:10.1007/s10953-0079182-x.

[30] D. Bordeaux, Gluconic Acid, Its Lactones , and SO2 Binding Phenomena in Musts from Botrytized Grapes, J. Agric. Food Chem. 50 (2002) 6408-6412.

[31] K. Igarashi, M. Samejima, K.E. Eriksson, Cellobiose dehydrogenase enhances Phanerochaete chrysosporium cellobiohydrolase I activity by relieving product inhibition., Eur. J. Biochem. 253 (1998) 101-6. http://www.ncbi.nlm.nih.gov/pubmed/9578466.

[32] Z. Yue, W. Bin, Y. Baixu, G. Peiji, Mechanism of cellobiose inhibition in cellulose hydrolysis by cellobiohydrolase., Sci. China. C. Life Sci. 47 (2004) 18-24. doi: $10.1360 / 02 \mathrm{yc} 0163$.

[33] Z. Fan, W. Wu, A. Hildebrand, T. Kasuga, R. Zhang, X. Xiong, Novel Biochemical Route for Fuels and Chemicals production from Cellulosic Biomass, PLoS One. 7 (2012) e31693. doi:10.1371/journal.pone.0031693.g001 10.1371/journal.pone.0031693.g002.

[34] A. Hildebrand, T. Schlacta, R. Warmack, T. Kasuga, Z. Fan, Engineering Escherichia coli for improved ethanol production from gluconate, J. Biotechnol. 168 (2013) 101-6. doi:10.1016/j.jbiotec.2013.07.033.

[35] S.H. Desai, C. a. Rabinovitch-Deere, Y. Tashiro, S. Atsumi, Isobutanol production from 
cellobionic acid in Escherichia coli, Microb Cell Fact. 45 (2015) 1-10. doi:10.1007/s00253-013-5504-7.

[36] P. Baldrian, Fungal laccases - occurrence and properties., FEMS Microbiol. Rev. 30 (2006) 215-42. doi:10.1111/j.1574-4976.2005.00010.x. 


\section{Figure captions:}

Figure 1. Chemical structures of cellobiono- $\delta$-lactone and cellobionate, with relevant protons for the NMR analysis identified by color-coded symbols. Red square = internal anomeric proton for cellobiono- $\delta$-lactone; purple circle $=\mathrm{C}$ - 2 proton for cellobiono- $\delta$-lactone; blue diamond $=$ internal anomeric proton for cellobionate; green triangle $=\mathrm{C}-2$ proton for cellobionate. Figure adapted from [36].

Figure 2. Proton NMR spectra for cellobionic acid and cellobiono- $\delta$-lactone at various $\mathrm{pH}$. Figure $2 \mathrm{~A}$ shows the full spectra at varying $\mathrm{pH}$. Figure $2 \mathrm{~B}$ is the spectra zoomed in at $4.58 \mathrm{ppm}$, corresponding to the internal anomeric proton of cellobiono- $\delta$-lactone. Figure $2 \mathrm{C}$ is the spectra zoomed in at $4.28 \mathrm{ppm}$, corresponding to the C-2 proton of cellobiono- $\delta$-lactone. The green triangles and blue diamond correspond to the C-2 proton and internal anomeric proton of cellobionate, respectively.

Figure 3. A) cellobionate inhibition of $\mathrm{CBHI}$ at $\mathrm{pH} 4$ or 6 ; B) cellobiose inhibition of $\mathrm{CBHI}$ at $\mathrm{pH} 4$ or 6 ; C) cellobionate inhibition of $\mathrm{CDH}$ at $\mathrm{pH} 4$ or 6 ; D) cellobiose or cellobionate inhibition of Accelerase 1500 atpH 5. At pH 4, the equilibrated solution of cellobionate is approximately $5.4 \%$ cellobiono- $\delta$-lactone and $94.6 \%$ cellobionate. At $\mathrm{pH} 6$, the equilibrated solution of cellobionate is entirely in the acid form. Error bars represent the standard deviation of reaction triplicates.

Figure 4. Avicel hydrolysis by CBHI with and without inhibitors present. Errors bars are the standard deviations of triplicate measurements.

Figure 5. Double-reciprocal plots showing competitive inhibition of CBHI with pNPL as the substrate for A) cellobionate and B) cellobiose, and C) cellobionate inhibition of CDH with 
cellobiose as the substrate. D) Kinetic constants, given in $\mathrm{mM}$, of CBHI inhibition by cellobiose and cellobionate and $\mathrm{CDH}$ inhibition by cellobionate. 
Table 1. Relative percent of cellobiono- $\delta$-lactone and cellobionic acid. The percentage was calculated based on the ratio of the peak areas for the C-2 protons and internal anomeric protons, as determined from the spectra in Figure 2. Standard deviations are from duplicate measurements.

\begin{tabular}{lcc}
\hline & \multicolumn{2}{c}{ RELATIVE \% } \\
& cellobiono- $\delta$-lactone & cellobionic acid \\
\hline $\mathrm{pH} 4$ & $5.4 \pm 0.2$ & $94.6 \pm 0.2$ \\
$\mathrm{pH} 5$ & $1.9 \pm 0.2$ & $98.1 \pm 0.2$ \\
$\mathrm{pH} \mathrm{6}$ & $0.0 \pm 0.0$ & $100.0 \pm 0.0$ \\
$\mathrm{pH} \mathrm{7}$ & $0.0 \pm 0.0$ & $100.0 \pm 0.0$ \\
$\mathrm{pH} 8$ & $0.0 \pm 0.0$ & $100.0 \pm 0.0$
\end{tabular}

Table 2. Kinetic constants, given in $\mathrm{mM}$, of $\mathrm{CBHI}$ inhibition by cellobiose and cellobionate at pH 5 with pNPL as the substrate, and CDH inhibition by cellobionate at pH 5 with cellobiose as the substrate.

\section{CBHI kinetics}

\begin{tabular}{lccc} 
Parameter & value & 95\% confidence interval & $R^{2}$ \\
\hline $\mathrm{K}_{\mathrm{m}}$ & 1.003 & $4.79 \mathrm{E}-02$ & 0.998 \\
$\mathrm{~K}_{\mathrm{i}}$ - cellobiose & 0.052 & $5.50 \mathrm{E}-06$ & 0.992 \\
$\mathrm{~K}_{\mathrm{i}}$ - cellobionate, equilibrated & 1.184 & $2.38 \mathrm{E}-05$ & 0.981 \\
\hline \multicolumn{2}{c}{ CDH kinetics } \\
Parameter & value & 95\% confidence interval & $R^{2}$ \\
\hline
\end{tabular}




\begin{tabular}{lccc}
$\mathrm{K}_{\mathrm{m}}$ & 0.172 & $2.90 \mathrm{E}-02$ & 0.932 \\
\hline $\mathrm{K}_{\mathrm{i}}$ - cellobionate, equilibrated & 0.732 & $1.97 \mathrm{E}-04$ & 0.941 \\
\hline
\end{tabular}


Figure 1

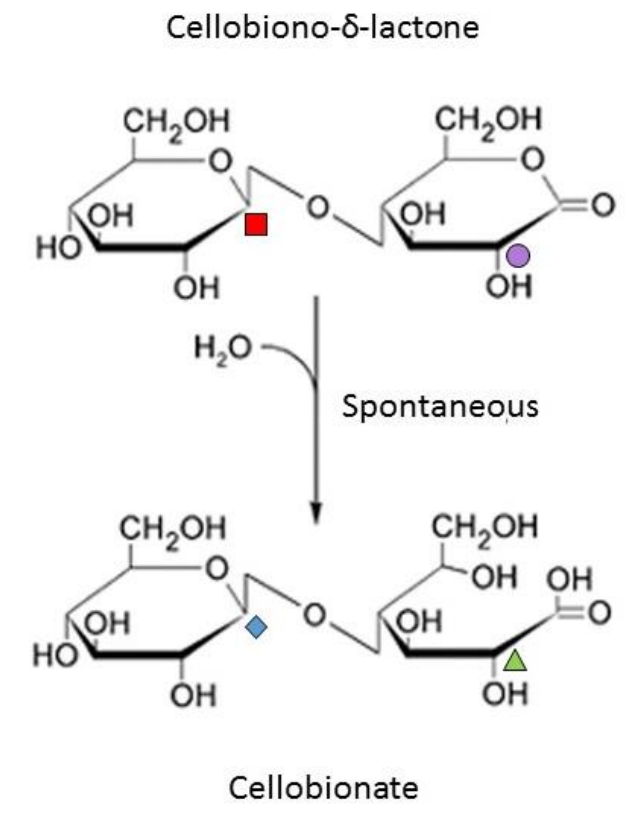


Figure 2
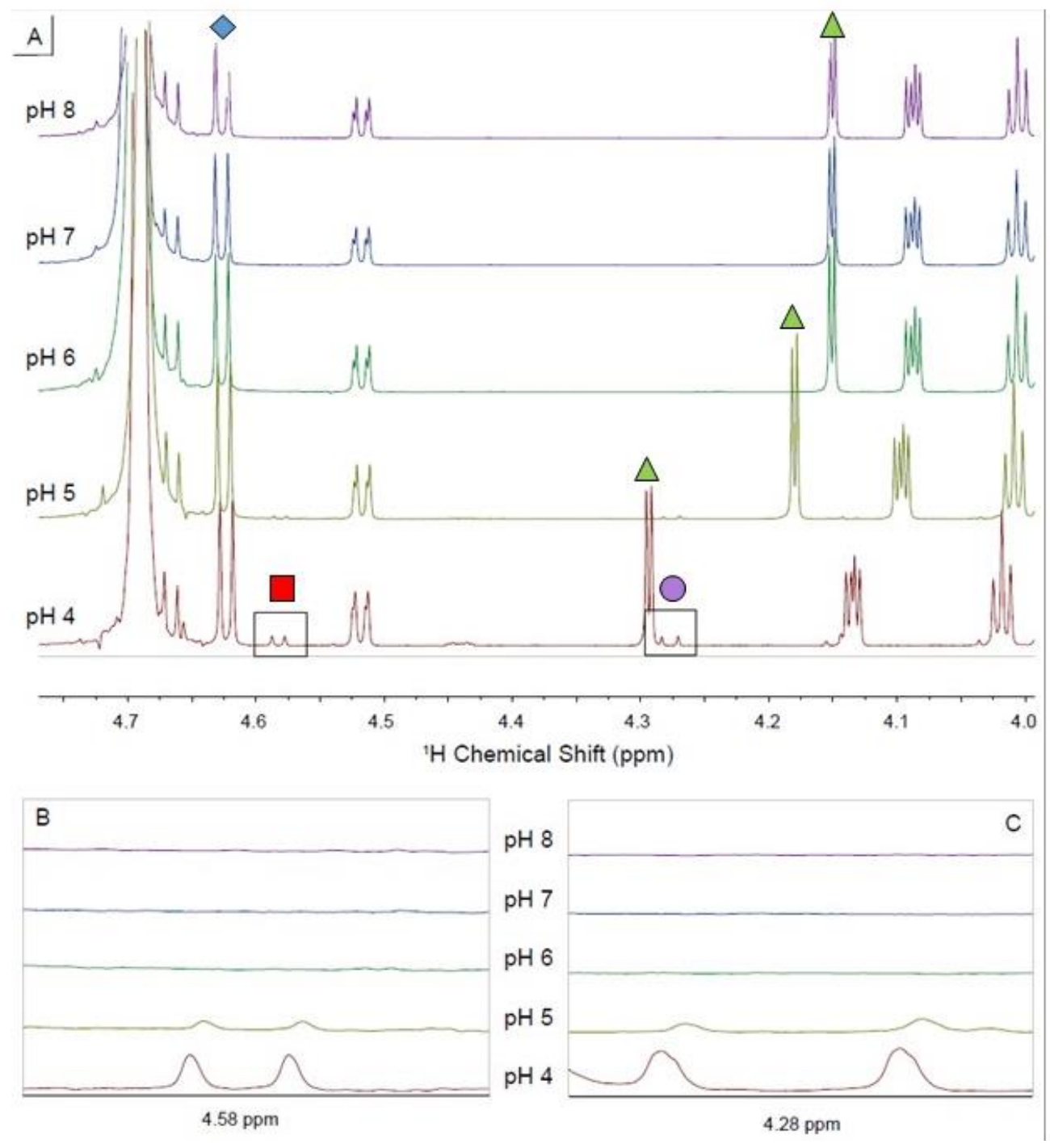
Figure 3

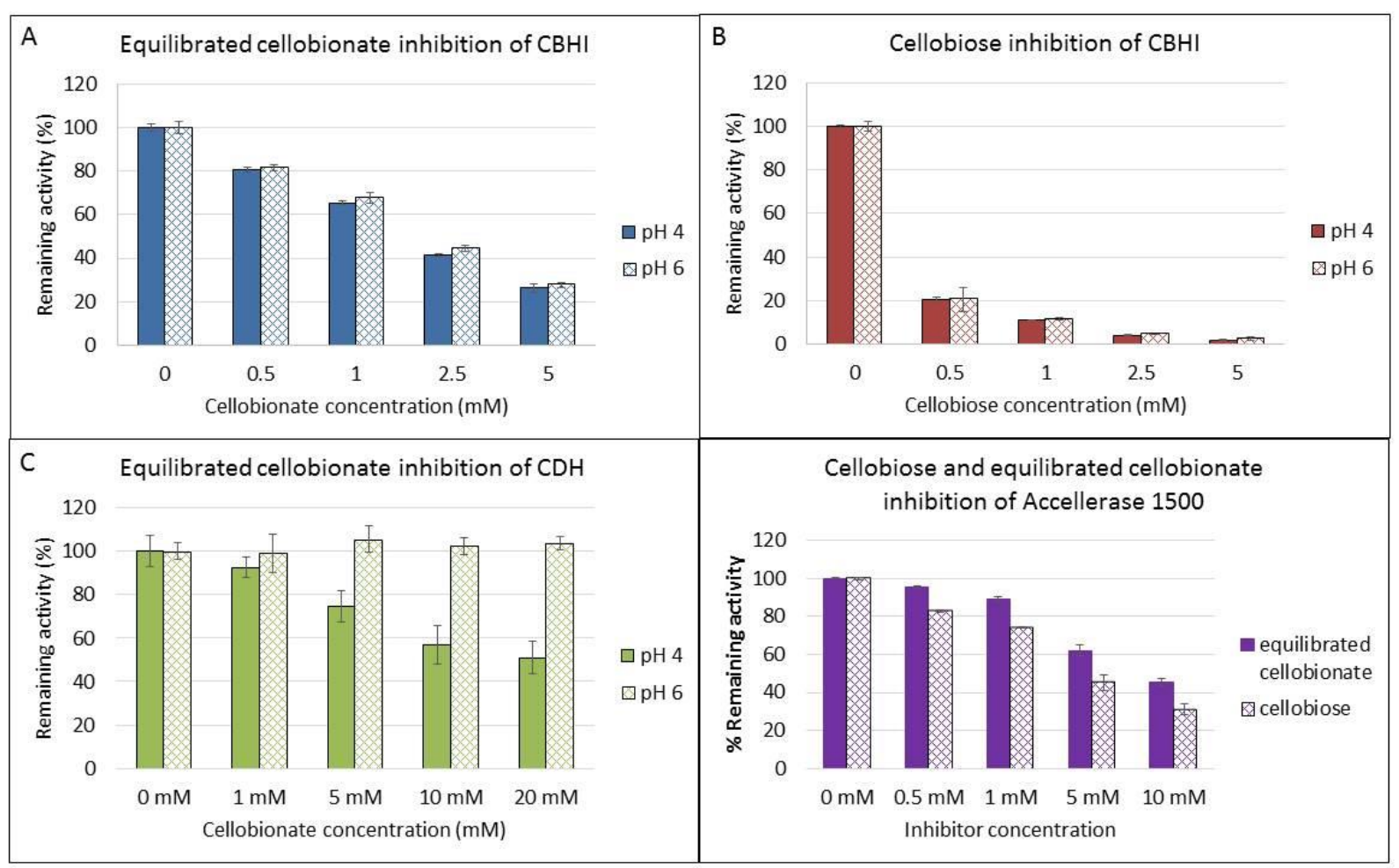


Figure 4

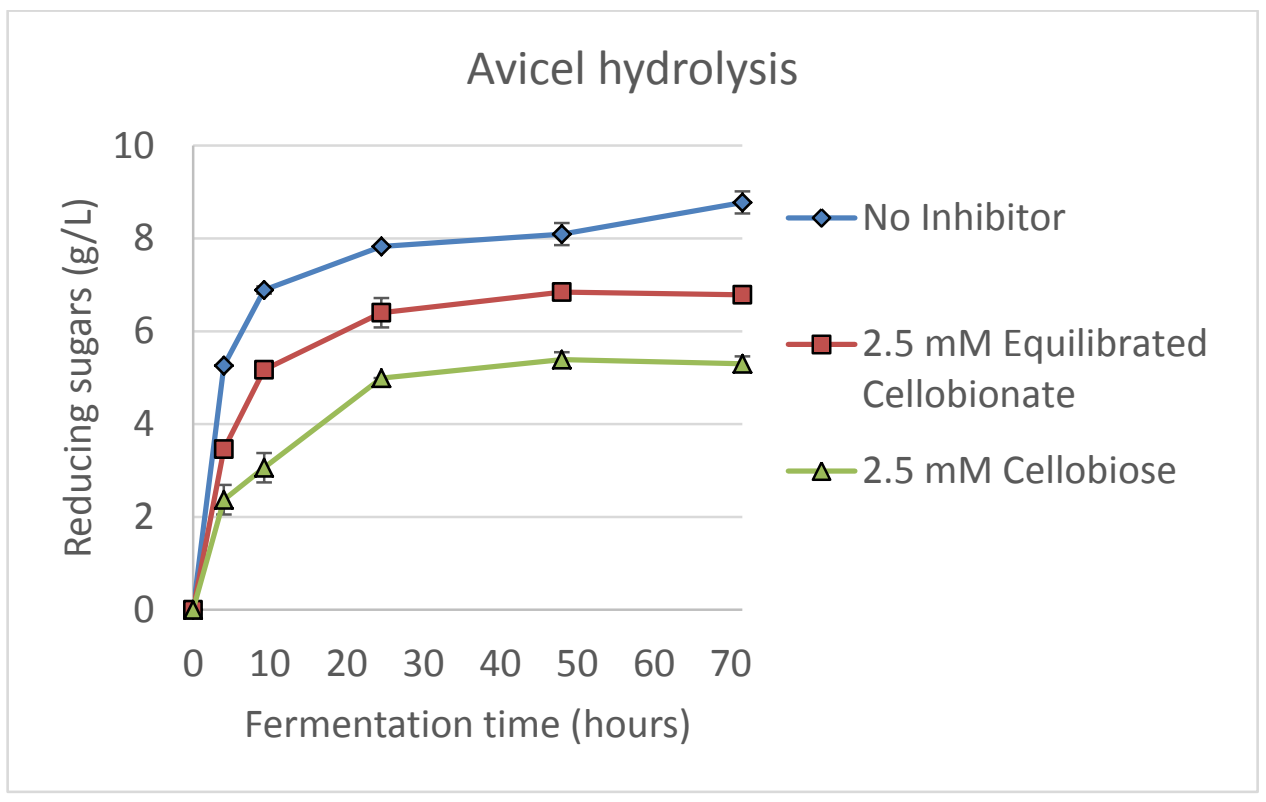


Figure 5

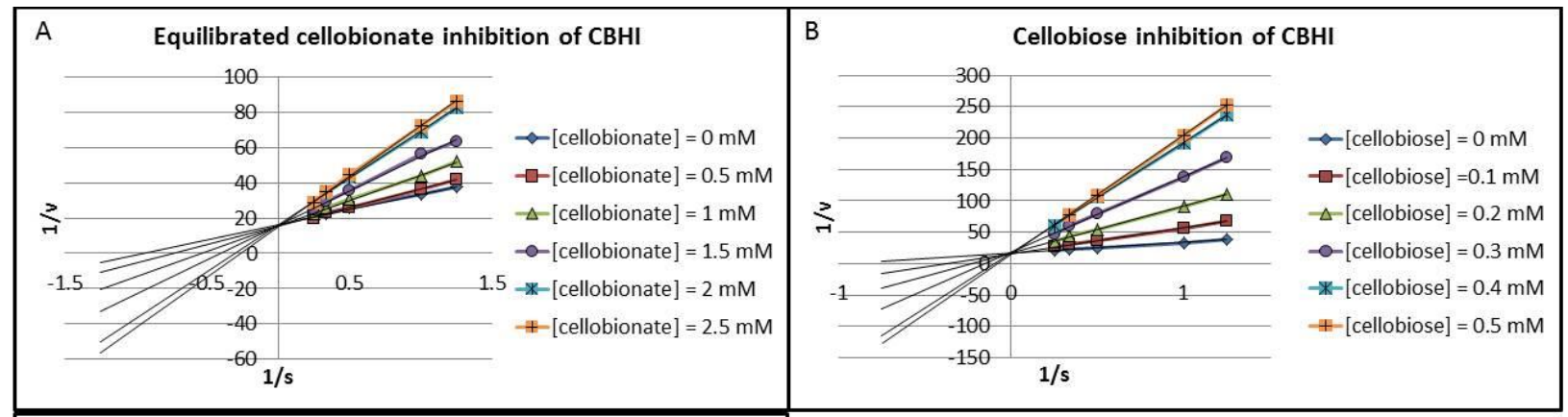

\begin{tabular}{lll}
\hline & Equilibrated cellobionate inhibition of $\mathrm{CDH}$ \\
250 & 200 & \\
\hline
\end{tabular}




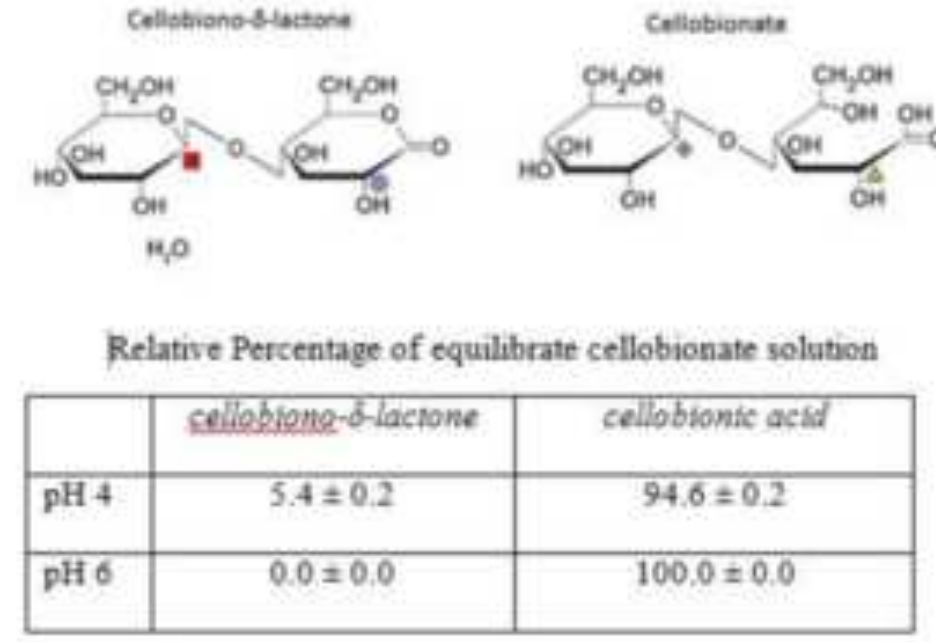

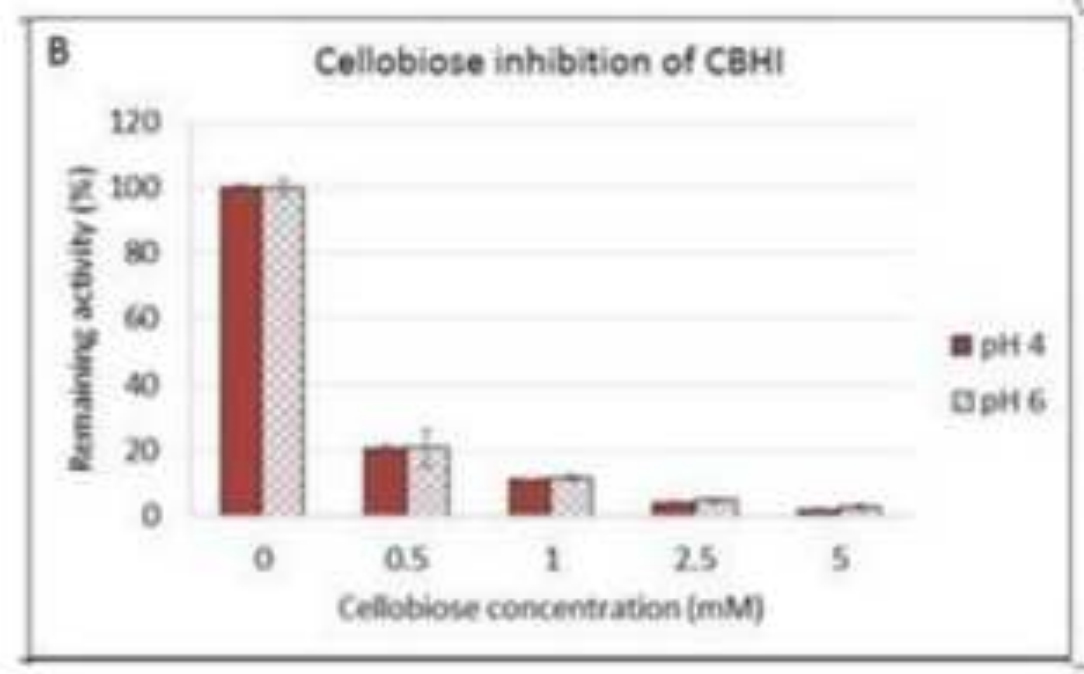

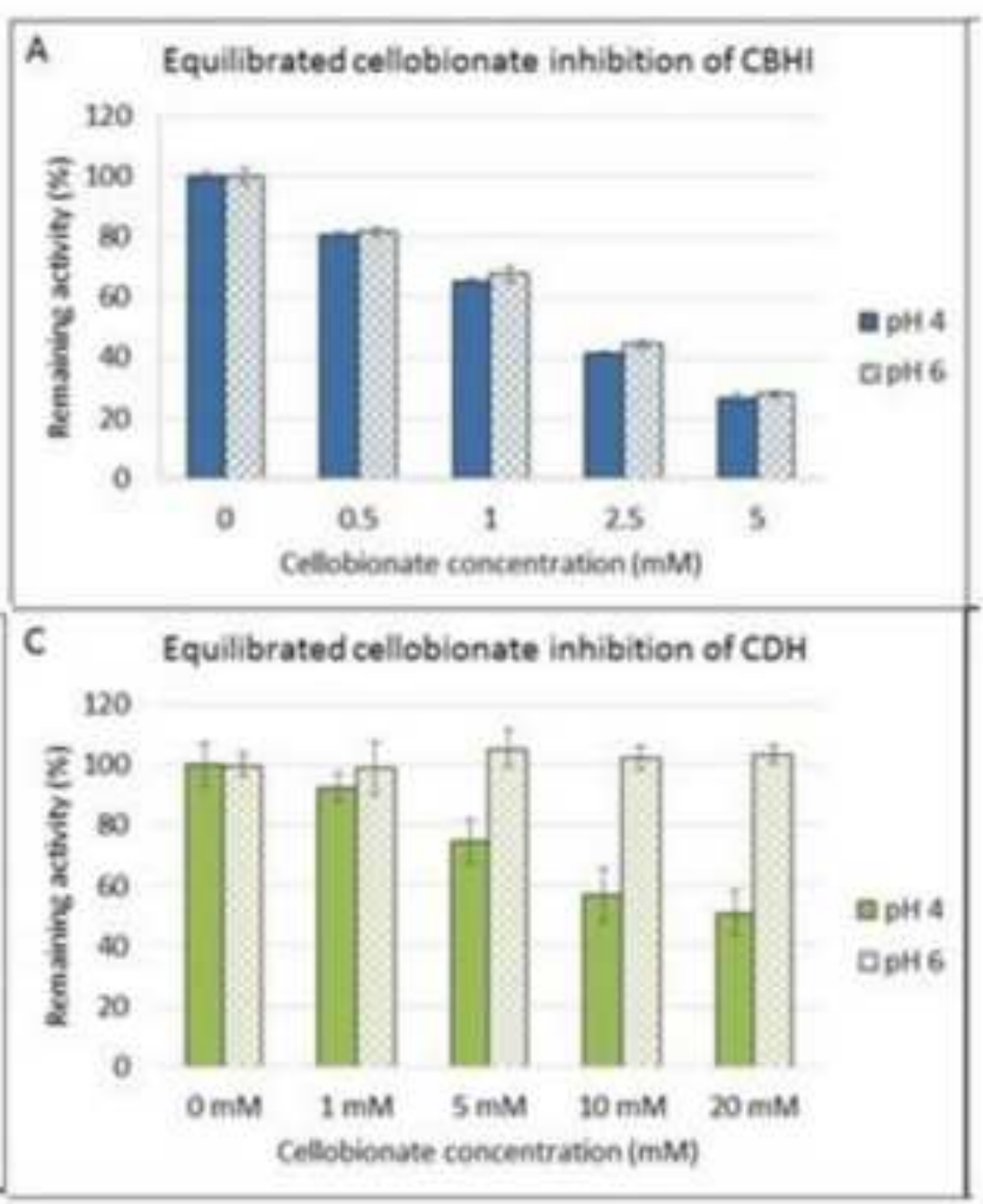

